\title{
Belphégor
}

Littérature populaire et culture médiatique

$17 \mid 2019$

Mutations des légitimités dans les productions

culturelles contemporaines

\section{Le chercheur face au fandom de SF : retour sur le fil $\mathrm{M}$}

\section{Simon Bréan et Irène Langlet}

\section{(2) OpenEdition}

Journals

Édition électronique

URL : https://journals.openedition.org/belphegor/1647

DOI : $10.4000 /$ belphegor. 1647

ISSN : 1499-7185

Éditeur

LPCM

Référence électronique

Simon Bréan et Irène Langlet, «Le chercheur face au fandom de SF : retour sur le fil M », Belphégor [En ligne], 17 | 2019, mis en ligne le 03 septembre 2021, consulté le 03 septembre 2021. URL : http:// journals.openedition.org/belphegor/1647 ; DOI : https://doi.org/10.4000/belphegor.1647

Ce document a été généré automatiquement le 3 septembre 2021.

\section{(c)}

Belphégor est mis à disposition selon les termes de la Licence Creative Commons Attribution - Pas d'Utilisation Commerciale - Pas de Modification 4.0 International. 


\title{
Le chercheur face au fandom de SF : retour sur le fil $\mathrm{M}$
}

\author{
Simon Bréan et Irène Langlet
}

Dans son versant américain, la littérature de science-fiction s'est très tôt accompagnée d'un discours critique endogène, celui des fans s'exprimant dans les courriers des lecteurs des revues, puis dans des publications d'amateurs, les «fanzines» dont le modèle s'est exporté dans bien d'autres domaines, et enfin dans les textes critiques et encyclopédies bâties par des fans de la première heure ${ }^{1}$. Cette dynamique s'est vigoureusement exprimée dans le champ littéraire français aussi, dans les colonnes de Fiction d'abord, puis dans des sommes critiques régulièrement mises à jour ${ }^{2}$. Ces ouvrages nourris de passion et de connaissances spécialisées ont longtemps fait référence, et certains restent encore indispensables aux chercheurs. Chacun d'eux porte néanmoins la marque très forte de la subjectivité de leur auteur.

La dynamique de création, d'accumulation, de diffusion et de partage d'images propre à la science-fiction n'est sans doute pas sans rapport avec la vigueur polémique des prises de position des acteurs de ce domaine, en particulier autour de questions de délimitation du corpus : la définition de la science-fiction est presque devenue un sousgenre littéraire en soi, et chacun a la sienne. Farouches technophiles, les fans de SF ont également été parmi les premiers à investir les supports numériques, dans des usenets comme fr.rec.arts.sf, des listes de diffusion comme SF-Franco, puis des forums spécialisés, tels que celui du Cafard cosmique ou celui d'ActuSF. C'est à partir de ce dernier que nous proposons de réfléchir à l'érudition des amateurs en science-fiction, en particulier en nous fondant sur le cas extraordinaire d'un fil de discussion alimenté entre le 28 octobre 2009 et le 17 octobre 2010. Intitulé « Du Sense of Wonder à la SF métaphysique » par celui qui l'a ouvert, Serge Lehman ${ }^{3}$, ce fil (surnommé « fil M » par les amateurs) stabilisé à 755 pages et plusieurs millions de signes ${ }^{4}$ tire son origine d'un affrontement polémique entre Lehman et Roland C. Wagner ${ }^{5}$. Dans la préface d'un recueil de nouvelles censé rassembler le meilleur de la science-fiction française, Retour sur l'horizon, paru en 2009 chez Lunes d'Encre, Serge Lehman a avancé une nouvelle interprétation de l'histoire de la science-fiction, en insistant sur l'importance des 
écrivains français d'anticipation, et en faisant de la métaphysique une "variable cachée » expliquant l'échec de la science-fiction en France. Cette littérature aurait été mal reçue car victime du refus de la métaphysique par les élites françaises. Roland C. Wagner a ensuite déplacé en partie le débat sur le Web : considérant l'introduction de la métaphysique comme un coup de force et comme une manière de réécrire l'histoire de la science-fiction pour se l'approprier, cet écrivain de la même génération que Lehman publie une critique virulente de la préface le 27 octobre 2009 sur le blog du Belial, éditeur de Bifrost, qui était alors (et demeure à ce jour) la grande revue spécialisée française. Il était prévu que ce texte soit repris dans son édition papier. Quelques heures plus tard, et avant donc la parution de la revue, le débat s'engage sur le forum d'ActuSF ${ }^{6}$.

3 Ce fil de discussion a attiré de nombreux intervenants, souvent des érudits majeurs, mais de toute façon des fans et connaisseurs. Outre Lehman et Wagner, Gérard Klein, Joseph Altairac, Olivier Paquet et plusieurs autres écrivains se sont investis dans la discussion avec des piliers de la communauté SF signant sous pseudonyme ${ }^{7}$. Même si certaines prises de position ont été d'une grande violence, nécessitant l'intervention des modérateurs ${ }^{8}$, l'ironie et la provocation n'ont pas pris le dessus sur l'argumentation et la démonstration, si bien qu'il représente après coup un modèle remarquable de la réflexion collective en science-fiction. Un modèle qui est peut-être aussi et surtout un moment de cette réflexion : les biais cognitifs propres aux réseaux sociaux s'y font déjà sentir, et la rencontre entre des opinions divergentes, maintenant rendue presque impossible par les bulles de filtre, ne s'y fait qu'avec bien des difficultés 9 . Néanmoins, ce moment, ce modèle, manifeste encore un espoir de rassembler tous les érudits de la science-fiction autour d'une agora commune.

4 La participation dont nous voulons rendre compte ici en témoigne. À l'époque, nous nous sommes en effet intéressés à ce fil avec des approches, des positions et donc des compréhensions radicalement différentes. D'un côté, un universitaire débutant (doctorant en 2009), érudit parmi les érudits, héritier du fandom, informé des autorités et des linéaments de la discussion, voire des identités cachées sous les pseudos (Simon Bréan). De l'autre, une universitaire installée (professeure en 2009), assez lectrice de science-fiction pour en avoir fait un axe de ses travaux, mais peu familière de ce fandom dont elle ne partage pas la culture (Irène Langlet). Aujourd'hui, nous avons en commun la méthode universitaire; nous voulons nous en servir ici pour éclairer des moments où elle se confronte à l'érudition des amateurs.

\section{Construction de savoir et déconstruction polémique (Simon Bréan)}

5 Au cours du fil «Du Sense of Wonder à la SF métaphysique », le désir de mettre en place des concepts et des outils pertinents est toujours allé de pair avec la remise en cause polémique de toutes les propositions. La mauvaise foi et la provocation n'ont pas été absentes: elles ont parfois provoqué la rupture totale de toute communication. Néanmoins, la configuration la plus productive n'a en fait jamais été la collaboration, mais bien la contestation systématique. Si d'autres espaces du forum contenant ce fil sont effectivement dévolus à la construction d'une connaissance commune («les infos sur »), celui qui accueille le fil (« Vos dernières lectures») est clairement placé sous le signe de la subjectivité et du goût personnel. L'implication dans ce fil reproduit donc le 
modèle d'une conversation, bien plus que celui d'une réunion de travail. Cette conversation associe les fans autant qu'elle les oppose, pour des enjeux de plusieurs natures : l'expression d'un discours exact sur l'objet de leur passion, bien sûr, mais aussi la manifestation d'une supériorité, intellectuelle, mémorielle, statutaire, ou encore la volonté de propager des thèses plutôt que de les mettre à l'épreuve, sans parler des biais individuels tels que refuser à un adversaire la satisfaction de lui donner raison. De ce fait, une forte polarisation se crée entre deux séries d'objectifs distincts : aboutir à un consensus, d'une part, faire triompher son point de vue individuel, d'autre part. Toutefois, c'est justement cette polarisation qui a permis d'alimenter si longtemps un fil de discussion aussi polémique : le désir de paraître plus pertinent que les autres a servi de moteur, parfois avec des explosions, tandis que la recherche d'un consensus servait d'idéal régulateur inaccessible ${ }^{10}$.

6 Je souhaiterais revenir ici sur un moment parmi d'autres de cette conversation à épisodes, au bout de trois mois d'échanges, mais vers le milieu du fil en termes de pages (de la p. 435 à la p. 443), qui correspond à près de 150000 signes publiés en environ 24h, soit l'équivalent de trois articles scientifiques de bonne taille ${ }^{11}$. Ce moment est relativement apaisé : Serge Lehman indique avoir abandonné son projet d'expliquer toute la science-fiction par la métaphysique, et la discussion tourne autour d'un autre projet, celui d'une histoire de la science-fiction. Je voudrais suivre l'examen collectif d'une théorie avancée au cours de la conversation, celle d'un «effet SF » dont la formulation est censée servir de consensus pour une définition de la science-fiction. Cela m'amènera à interroger la possibilité de construire un savoir partagé sur la science-fiction dans un tel contexte.

7 Serge Lehman présente ainsi, au sein d'un discours plus général, la notion qui donne ensuite prise à la discussion :

Oublions le merveilleux-scientifique et le sow [Sense of Wonder] et qualifions l'objet d'« Effet SF » sans en dire plus pour l'instant.

La proposition devient alors : je considère la SF sous l'aspect d'une expérience subjective, d'une position esthétique avec les émotions associées. Cette expérience, cette position, c'est « l'effet SF » et je me demande s'il ne peut pas nous fournir des éléments de définition. (p. 436, 20 janvier 2010 19h37)

Cette proposition d'un "Effet SF " s'inscrit dans la continuité d'une conversation bien antérieure, concernant le "Sense of Wonder", ce "sens de la merveille», ou de "l'émerveillement", qui fait partie des attributs les plus difficiles à cerner pour la science-fiction. Lehman lui-même l'associe à une logique de vertige, de décentrement de perspective, et en a fait précédemment un indice de la nature «métaphysique » des objets de la science-fiction. Ces derniers seraient alors à la fois ancrés dans un certain réel, et impossibles à comprendre tout à fait: c'est cela qu'il place au cœur de sa définition de la science-fiction, cette esthétique de l'excès rationnel, du "merveilleux scientifique », pour reprendre l'expression de Maurice Renard dont l'exégèse a lancé le fil « Du Sense of Wonder à la SF métaphysique » ${ }^{12}$.

9 Ainsi, l'expression « effet $\mathrm{SF}$ » naît de la nécessité de se débarrasser d'une terminologie devenue embarrassante, car trop battue en brèche. Après la métaphysique, dont le lien avec la science-fiction a été refusé en bloc par de nombreux participants, puis le "merveilleux scientifique», qui n'a pas convaincu de sa pertinence concernant la science-fiction contemporaine, c'est le caractère opératoire du "Sense of Wonder » qui se voit nié. Olivier Paquet (Erion) le trouve trop subjectif pour être discriminant, et donc pour se trouver au cœur d'une définition de la SF. Joseph Altairac (Lensman) 
souligne qu'il n'y a pas un Sense of Wonder, mais autant de variétés que de sous-genres de la science-fiction (p. 435, 20 janvier 2010 18h41).

L'« effet SF » part d'une logique de contournement, mais il ne s'agit pas d'une simple reformulation. C'est l'occasion pour Serge Lehman de proposer une mise au point sur sa théorie de la science-fiction, en profitant de la discussion pour tester de nouvelles propositions. D'une manière caractéristique des subtilités de sa posture argumentative, il s'abstient de signaler qu'en employant cette expression, il se réapproprie un titre très respecté des amateurs, L'Effet science-fiction, recueil de témoignages édité par les frères Bogdanoff, qui posait déjà la définition de la science-fiction en dehors des textes euxmêmes, en la situant chez ses observateurs (Bogdanoff, 1979).

Les arguments de Serge Lehman se déploient de la manière suivante. Il propose d'abord, de manière consensuelle, l'idée qu'un « effet SF » naît du paratexte, du contact cumulatif avec des indices génériques, " au contact du sigle et de son apparatus ». Puis il glisse rapidement vers une définition plus large, permettant d'identifier la sciencefiction sans ses indices génériques à un procédé stylistique qu'il dénomme la "métaphore réifiée ", ou la "réification des métaphores", dont il trouve un exemple adéquat chez Borges :

Ma réponse : ce qui produit l'Effet chez Borgès, c'est le fait que la proposition « l'univers est une bibliothèque infinie » est prise au pied de la lettre. Qu'elle n'est pas la métaphore inaugurale d'une fable sur la multitude des mémoires humaines, ou d'une allégorie sur le rôle du livre dans la civilisation mais une réalité concrète, matérielle, qui fonctionne comme telle sans intervention de l'auteur. L'univers est une bibliothèque infinie et le texte examine les conséquences logiques de cette proposition. Et c'est précisément cette position, exprimée à travers un certain usage du langage, qui produit l'Effet SF. (p. 436, 20 janvier 2010 19h37)

De là, Serge Lehman ajoute à sa tentative de clarification théorique un objectif externe : permettre l'inclusion de la science-fiction dans la littérature générale en montrant que des auteurs tels que Borges produisent un « Effet SF ». Il en profite pour réintroduire la métaphysique: la "métaphore réifiée » revient à rendre concrète une proposition métaphysique.

Or, ce flou, cette incertitude, c'est précisément le régime cognitif du métaphysique. Dire «l'univers est une bibliothèque infinie ", c'est une proposition métaphysique. Réifier cette proposition, la considérer sous sa seule acception concrète, matérielle, c'est la faire passer du métaphysique au physique - et c'est à cet instant que se produit l'Effet. (p. 436, 20 janvier 2010 19h37)

13 La posture rhétorique vise ici à faire admettre la pertinence d'ouvrir un espace de dialogue entre une science-fiction identifiée et une science-fiction "qui s'ignore »: établir une continuité entre un canon légitime et le genre à défendre, placée sous le signe d'un même rapport à un questionnement métaphysique, source de vertige conceptuel. L'ambition de cette proposition la rend quelque peu instable : beaucoup semble reposer sur la reconnaissance de cet "Effet SF », qui embrasse de nombreux aspects. En réaction, les débatteurs du fil se concentrent sur des points spécifiques, en particulier, le concept de «métaphore réifiée »"

14 Pour mettre à l'épreuve cet aspect de la théorie de Lehman, les uns et les autres s'appuient sur des exemples partagés, qui sont de deux natures : des exemples d'œuvres de référence, et des exemples construits pour l'occasion. Soleil vert propose ce soutien à la théorie de Lehman : 
"je n'ai pas compris ce qu'est une "métaphore réifiée" ".

Une expression prise au pied de la lettre : dans Océanique, l'immersion, ou plutôt le contact avec les organismes minuscules qui y résident, engendre un sentiment d'euphorie converti en béatitude religieuse (la religion est un sentiment océanique). (p. 439, 21 janvier 20103 h19)

Gérard Klein engage un micro-récit humoristique fondé sur la comparaison «La Terre est bleue comme une orange ".

À peine sorti du métespace, ayant fait un bond grossièrement évalué à vingt milliards d'années-lumière et s'étant donc échappé de la sphère d'observation de la Prime Galaxie, le navire se stabilisa en orbite autour d'un soleil dont les paramètres semblaient étrangers à ceux connus dans son univers d'origine.

Cette étoile rayonnait puissamment dans le bleu et les détecteurs de quarks et de wimpinos indiquaient que sa composition ressemblait à celle d'une orange.

Certes très cuite. (p. 439, 21 janvier 2010 2h27)

On trouve aussi un cas intermédiaire, qui est celui de l'œuvre de référence partiellement réécrite pour servir d'appui à la thèse. Ainsi, Serge Lehman s'appuie sur un roman de Gérard Klein, Les Tueurs de temps, pour développer quatre interprétations de l'expression "tuer le temps", en lien à chaque fois avec un type de récit. Ces cas se situent dans un spectre allant du plus réaliste au plus science-fictionnel, pour situer dans le dernier la réification de la métaphore. Je suis moi-même intervenu alors pour proposer un cinquième cas, qui faisait intervenir une métaphore réifiée selon une modalité de fantasy. J'ai continué par la suite en imaginant des manières de réifier des métaphores y compris en régime ontologique réaliste.

17 Joseph Altairac et Olivier Paquet continuent un dialogue parallèle avec Serge Lehman. Le premier remet en cause la pertinence de chercher à étendre cet «Effet SF » hors du domaine de la science-fiction, en critiquant l'usage d'exemples limites: "J'aime énormément Borges, mais la SF, elle existe pour moi sans aucun problème sans Borges.» (p. 443, 21 janvier 2010 16h02) Le second assène l'inadéquation de la réification des métaphores comme facteur de définition d'un genre jugé central pour la science-fiction, à savoir la «hard science », avant d'aboutir à une opposition frontale : «Que tu trouves des métaphores réifiées dans la SF, ok. Que tu trouves de la métaphysique dans la SF, ok. Mais que tu affirmes que les métaphores réifiées, le Sense of Wonder et la métaphysique DEFINISSENT la science-fiction, non. » (p. 443, 21 janvier 2010 16h12).

En définitive, la tentative de cristalliser un projet commun de légitimation autour d'une expression consensuelle, l'«Effet SF ", s'est heurtée aux mêmes lignes de clivage que le reste de la discussion, entraînant la disparition de l'expression dans le reste du fil, alors même que Serge Lehman poursuivait l'ambition dont elle avait été brièvement le nom. Néanmoins, ce clivage n'a pas empêché qu'entre dans la réflexion commune la notion de «métaphore réifiée »: parfois moquée, cette contribution de Lehman à la compréhension des mécanismes de la science-fiction refait surface à de nombreuses reprises, et sa résistance aux critiques garantit à terme sa pertinence heuristique. Le fil de discussion aura ainsi permis de tester l'efficacité de formulations et de démonstrations spécifiques.

19 Toutefois, ce qui a fait la force de ces débats est sans doute aussi ce qui en a empêché la fructification finale. Le dynamisme des échanges provient de conditions structurelles favorisant aussi les malentendus et les polémiques: les conversations croisées font entrer les participants dans des cercles vicieux d'arguments et de contre-arguments qui 
se répondent souvent mal; les délais entre les messages provoquent parfois un décalage très important entre une proposition et sa réfutation, au risque parfois que la proposition initiale ait déjà été amendée. Plus important encore, les participants sont réunis et divisés en même temps en raison d'une différence fondamentale d'objectifs : au-delà du désir de tenir un discours cohérent sur la science-fiction, se joue la question d'un magistère intellectue ${ }^{14}$. En effet, l'un des enjeux de la discussion dépasse l'élaboration théorique. Le discours de Serge Lehman le porte vers un « extérieur » qui est le domaine des institutions littéraires générales, au-delà même des cadres universitaires où il a déjà reçu bon accueil ; dans le cadre du forum, il semble dès lors aspirer à tenir le rôle d'un représentant de toute la science-fiction française; et il est accusé de chercher dans la conversation moins une vérité, que la validation de sa vérité. Cela le conduit en général à esquiver les arguments les plus percutants, en changeant d'argument sans concéder d'avantage à ses contradicteurs. Inversement, les autres participants cités ici lui contestent, chacun à un degré différent, cette autorité ; mais ils se satisfont trop souvent de la contradiction, comme si déconstruire le système de Lehman suffisait à établir une théorie de la science-fiction.

Pour remarquable que soit l'exemple du fil «Du Sense of Wonder à la SF métaphysique ", il fait surtout apparaître la difficulté de construire un savoir commun du fait d'un programme esthétique divergent, qui a opposé l'ambition totalisante d'un Serge Lehman décidé à diffuser sa définition de la science-fiction dans le domaine littéraire à la précision souvent très exigeante des fans acharnés à défendre des perspectives centripètes pour leur genre de prédilection.

\section{De la référence à la posture : l'autorité en jeu (Irène Langlet)}

21 À ce « retour dans le fil $\mathrm{M}$ » d'un vétéran ayant vécu la bataille de l'intérieur, j'ajoute à présent le « retour vers le fil $\mathrm{M}$ » d'une lectrice doublement extérieure : parce que hors du fandom à l'époque ${ }^{15}$, et parce que je n'y ai donné aucune contribution écrite. C'est l'occasion de revivre des impressions restées vives, face aux facettes du fil M.

La plus spectaculaire est ce régime de parole où, tout à la fois, l'échange semble déréglé et le savoir ne se fixe pas. Dans ce «magistère » identifié plus haut, toutes mes prises ordinaires de critique universitaire - les sources, références et autorités, constamment évoquées - ne sont déterminantes ni en tant qu'état de la question, ni en tant que mention d'attribution (ainsi des Bogdanoff, auteurs non crédités de la fameuse expression "effet science-fiction »), ni en tant qu'arguments d'autorité. Dans une des premières passes d'arguments, Wagner atténue celui de Lehman, ainsi que sa référence, en la désignant ainsi : « l'extrait de la lettre de GPW [G. Peyton Wertenbaker] que tu as cité je ne sais plus où », et réplique à cet argument par une citation de Gary Westfahl : "Voici maintenant ce que Gary Westfahl écrit au sujet de "Twilight", de John W. Campbell (publié sous le pseudonyme de Don A. Stuart en 1934)»(p. 159, 7 décembre 2009 17h34). À ce faisceau d'indices de précision, Lem répond avec la même désinvolture : "Qu'écrit-il ? Si j'ai bien compris, que (...) » puis il efface complètement (et avec la même désinvolture subjective) l'autorité de l'argument (la position de Westfahl comme érudit reconnu internationalement): «Pourquoi pas? Mes souvenirs de lecture de Campbell sont assez lointains mais ça me paraît plausible.» (p. 160, 7 décembre 2009 18h57). 
elques jours plus tard (le vendredi 11 décembre), «Lem » d'un côté (le pseudo de Lehman sur le forum), Wagner de l'autre, claquent successivement la porte et déclarent se retirer de la discussion. Tout se passe comme si les éléments de consensus, par exemple sur la métaphore réifiée, n'étaient que des retombées hasardeuses de la lutte pour le pouvoir épistémologique et discursif dont le forum est le théatre, prenant à rebours le processus académique (le mien, en l'occurrence) où est censé se passer le contraire : accumuler les apports critiques, en se résignant (si possible en gardant le sens de l'humour) aux luttes de pouvoir qui en sont les retombées inévitables. La lecture du fil $\mathrm{M}$, aujourd'hui comme hier, frappe surtout dans cet aspect, couplé à la prolifération de la parole. Cette dernière s'impose aujourd'hui par la masse de son volume désormais statique ; en direct, entre 2009 et 2010, la dynamique de ce processus rendait totalement impossible un suivi intégral des discussions, à moins d'y passer ses jours et ses nuits :

$\mathrm{Au}$ fur et à mesure qu'enfle le fil, les nouveaux arrivants se trouvent condamnés à des heures de lecture préalable ou à risquer de réutiliser un argument déjà disqualifié. Les messages peuvent parfois être très longs à écrire, si bien que des réponses s'intercalent, provoquant des bifurcations. Cela rend encore plus difficile la relecture de ce fil, qui semble parfois très chaotique. (Bréan, 2013).

Pour ma part, j'avais donc fini par n'y venir que de temps en temps, pour voir si « ça » continuait, avec à chaque fois un sentiment de surprise (oui, ça continuait) et de déroute devant les développements imprévus, tout cela mêlé à une croissante et troublante familiarité face à des voix dont je reconnaissais désormais les marottes, affinités ou hostilités, sans toutefois y être initiée. Associée à l'absence de références, cette prolifération mène à une paradoxale enclosure de la discussion, qui ne se nourrit guère des autres débats précédents ou parallèles (et ne les nourrit pas non plus).

Lorsque les références apparaissent, elles ne se laissent elles-mêmes saisir qu'au prix d'une sorte de jeu de piste pour la néophyte (et de recherches assidues pour l'universitaire qui voudrait contextualiser ces travaux : j'y reviendrai). On a vu plus haut comment une référence allographe était assez promptement réduite à une impression subjective, ce qui désactive le possible débat de savoirs savants qui aurait pu prendre place alors sur le forum. Les références autographes le désactivent d'une autre manière, qui tient plus du feuilleton, ou du storytelling d'autorité. « Lem » y construit une posture d'intellectuel à la fois détaché et assidu, erratique mais souterrainement constant, qui ne manque pas d'être relevée par un lecteur : «Lem (...) Tu es un peu des fois vachement difficile à suivre." (Le_Navire, p. 268, 18 décembre, 2009 22h14). Je voudrais revenir sur cet épisode des dernières semaines de l'année 2009, c'est-à-dire plutôt peu de temps après le début du fil (si l'on considère sa masse et sa durée totale), mais plutôt longtemps après (si l'on s'en tient à la durée moyenne d'un thread de forum sur internet, autrement dit si l'on se remet dans l'état d'esprit des participants du moment). La discussion vit alors l'un de ses moments-charnières: les premiers échanges sur le Sense of Wonder et la métaphysique ont déjà été déplacés vers la question d'une écriture de la $\mathrm{SF}$, puis d'une histoire revisitée. C'est le moment où les références à Wertenbaker et à Westfahl sont bottées en touche ; c'est aussi un moment marqué par l'un des éclats de ce « moteur à explosions » du forum.

Le cheminement de la référence autographe prend donc place dans un contexte dramatisé ; elle y acquiert des fonctions pragmatiques d'arbitrage. Or la première occurrence est étonnamment désinvolte: "Dans un papier (ne sais plus lequel, La physique des métaphores, je crois), j'avais de mon côté choisi de prendre l'expression d'un 
bloc : sense-of-wonder » (p. 37, 13 novembre, 2009 18h29). Le post de Lem commence directement par cette référence, et se poursuit par « c'est aussi pour cette raison que je considère Renard comme le vrai premier théoricien de la sf ». L'évocation du « papier $^{16}$ » n'est désinvolte qu'en apparence. Elle sert, en réalité, de garantie dans le débat. C'est alors qu'intervient l'échange sur Wertenbaker et Westfahl (début décembre 2009), dans une dynamique plutôt savante (c'est-à-dire familière pour moi) où les références s'appellent les unes les autres et règlent l'échange d'arguments sur leur autorité ou leur réfutation. On a vu que ce «moment savant » tourne court, entrainant peut-être le fil entier vers une fin abrupte. Mais Lem revient sur ses pas, et sur la référence de ses travaux, le 14 décembre, dans un geste qui dramatise nettement l'adieu dès son incipit :

Une résurgence provisoire pour ne pas laisser les derniers messages sans réponse. Mon post lapidaire de vendredi avait pour but d'endiguer le déluge de $\mathrm{mp}^{17}$ injurieux (...). Mais je m'en serais voulu de conclure aussi sèchement. (p. 208, 14 décembre 2009 8h03)

Le reste du post est à l'avenant: il multiplie les phrases conclusives, les formulations apaisantes, les concessions argumentatives ou personnelles («Dans ce cas, oui, d'accord, je comprends et j'assume» ou «Effectivement, j'ai eu tort (...) J'aime en découdre. Dont acte »), et il se clôt sur un envoi sans ambiguïté : " Merci à tous ceux qui ont contribué au fil (...) Le débat reprendra un jour ou l'autre, sous une forme ou sous une autre, ici ou ailleurs ». C'est dans ce cadre, qui congédie le débat, que la référence autographe se précise : «J'ai écrit pas mal de choses là-dessus depuis 2001 (La physique des métaphores, La légende du processeur d'histoire, Par-delà le vortex, Un souvenir du sac à charbon...) et je ne désespère pas de produire un jour une représentation satisfaisante. » En outre, ni le contexte, ni la teneur de ces écrits ne sont précisés, ce qui leur donne le statut de garanties globales d'autorité : j'ai écrit des choses, je vais en faire un livre, donc je ne parle pas sans savoir. D'une manière qui pourrait correspondre, toutes proportions gardées, aux usages du savoir savant, le débat est ainsi reporté non pas tant à un "ailleurs " qu'à un «demain ", quand les lecteurs se seront appropriés les écrits de celui qui parle, ou quand ce dernier aura publié sa somme. La posture d'autorité de ce post conclusif se manifeste ainsi doublement : un savoir validé par les publications étaye une attitude conciliante dans le débat.

Mais c'est au prix de ce qui peut passer pour une certaine discourtoisie, voire condescendance, étant donnée la nature des échanges qu'on a définie plus haut. De fait, les participants n'ont pas tous interprété la posture de Lem comme bienveillante, et l'échange s'est poursuivi (décrédibilisant quelque peu la dramatisation de l'adieu). "Le_Navire", en particulier, regimbe; sa protestation éclaire le cheminement souterrain de Lem, et l'échec de ce qui est désormais désigné comme un storytelling rhétorique passablement irritant, notamment par sa posture d'autorité artificiellement minimisée :

Lem je rejoins ce que disaient MF et Aldaran (et qui fut la cause de ma brusque montée de tension) Tu es un peu des fois vachement difficile à suivre.

(...)

Tu arrives avec une théorie, exposé [sic] dans une préface publiée.

Tu mets des plombes à la poser, le temps qu'on se soit tous entremordus le derrière aux moins quinze fois.

Tu justifies son existence en parlant du déni et de l'envie qu'on a d'en sortir.

Puis tu nous dis qu'en fait, ce qui t'intéresse c'est une histoire revisitée de la SF.

Et puis quand on t'interroge sur l'impact que tu penses qu'un tel travail puisse avoir 
dans la remise en cause du déni, tu me ponds : «ça n'a pas d'importance ». (p. 268, 18 décembre 2009 22h14) de préciser ses sources vis-à-vis d'un public guère convaincu de son magistère - ou plutôt, de façon plus intéressante pour notre propos, guère convaincu par cette manière de l'amener. Les érudits amateurs n'ont pas considéré le savoir savant comme une preuve suffisante; la protestation de Le_Navire dit aussi que son arrivée progressive, au fil de la discussion, a composé un véritable feuilleton, qui raconte une mauvaise histoire. La réponse de Lem montre qu'il perçoit très bien l'adresse à l'écrivain qui se cache dans cette protestation, puisqu'il se place sur le plan de l'écriture :

Il y a une phrase de Michel Foucault que j'ai déjà citée deux ou trois fois. «Si je savais ce que je pense avant de l'écrire, je ne l'écrirai [sic] pas.» C'est un peu comme ça que ça se passe, pour moi. (p. 269, 18 décembre 2009 22h44)

Il n'en reste pas à cette pirouette et se positionne sur au moins deux autres fronts : d'une part, il développe les travaux publiés; d'autre part, il commence (encore très implicitement) à admettre une ambition totalisante et un magistère affirmé. Ainsi, il en précise non seulement toute une série d'autres titres, mais les contenus, par exemple : «La préface que tu évoques est le dernier d'une série de textes également publiés où j'ai abordé le problème de la métaphore (Vers la fiction analogique, la physique des métaphores) (...) avec une inflexion sur la religion à un moment (Un souvenir du sac à charbon) (...) ». Lem conclut sur son ambition : «ce que j'ai réalisé pendant ce fil, c'est que j'étais peut-être effectivement en train de sortir des articles et des monographies pour commencer à chercher une synthèse ", en admettant sotto voce qu'elle n'est pas modeste : «Et franchement, à la question "quel effet escompté ?", tu imagines ce que j'aurais essuyé ici si j'avais répondu : “décisif"... » Mais le résultat est loin d'être une stabilisation de l'échange, au point que deux jours plus tard (le 20 décembre à 17h12) Lem revient à la charge et copie-colle dans le forum, in extenso, toute la partie 2 de sa conférence "La légende du Processeur d'histoires », en donnant sa référence de façon relativement précise (mais sans la date). Le cheminement autographe trouve là son point d'aboutissement : d'abord désinvolte («je ne sais plus où »), Lem fournit ensuite des références sélectives, puis donne une liste très fournie et commentée, et enfin s'autocite in extenso. Cela représente une masse considérable de texte dans le cours du fil, qui coupe court à la circulation des prises de parole : la preuve textuelle de son autorité s'en retrouve assénée avec fracas. Mais ça ne suffit toujours pas, et l'on peut prendre la mesure de l'agacement ressenti par Lem dans une réaction qu'il poste environ deux heures plus tard, le même jour :

ma première note critique sur la SF date de 83 , à la radio. Mon premier papier professionnel de 90. Les linéaments de cette théorie remontent à 2000. En tout, j'ai dû publier quelque chose comme deux cents textes sur la question. L'idée que je me sois interrogé, que j'aie soigneusement choisi les critères sur lesquels je souhaite travailler t'est-elle impossible à concevoir ? Tu as vraiment l'impression que je suis un amateur enthousiaste qui tombe dans le premier panneau venu parce qu'il ne connaît pas assez son sujet? (p. 277, 20 décembre 2009 18h59)

L'agacement naît de l'absence de reconnaissance; en contexte, il naît aussi de l'inefficacité de son procédé (la référence autographe), qui est le versant propre à Lem de l'impression ressentie aussi par la lectrice que j'étais : le savoir ne semble pas se fixer, les références ne sont pas enrôlées dans un processus de cumul des savoirs et de progression documentée et argumentée vers un consensus. On peut, et on doit 
également le replacer dans l'économie discursive particulière du forum: certes, le dimanche 20 décembre après-midi, lorsqu'elle est postée sur le forum, la conférence de Lem n'est commentée par personne. Mais on peut facilement imaginer les conditions de sa réception : quelques centaines de personnes, un dimanche peu avant de dîner, sont connectées en même temps à un forum où une dizaine d'entre elles débattent avec passion d'un problème littéraire. La disponibilité de chacun n'est pas toujours aussi grande; les questions-réponses, les rebonds courts, sont préférés à la lecture du morceau conséquent posté par Lem, qui ralentirait la discussion. Le geste de Lem se comprend dès lors comme au moins ambigu: est-ce la maladresse d'un auteur réclamant un peu d'attention et de reconnaissance pour son travail ? Ou bien est-ce une manière pour lui de chercher à éteindre la discussion?

Vue depuis ma position, c'est-à-dire plutôt celle de l'institution, l'aventure du fil M fait réfléchir aussi bien au savoir savant qu'à l'érudition des amateurs, et à leur zone de contact qui n'a rien de simple, ni dans la sociabilité à mettre en œuvre, ni dans la stabilisation des travaux accomplis. Car le récit de ce moment autocitationnel du forum est aussi celui d'un échec de Lehman, qui tient à la posture intermédiaire de «savant subjectif » revendiquée par l'écrivain. Or le fil M permet de voir que cet entre-deux ne convainc pas plus aisément en régime amateur qu'en régime savant. Lensman se plaint d'un «basculement dans le monde pédant (désolé si ça fait de la peine à Lem (...)) » (p. 144, 5 décembre 2009 14h12). Plus tard, lorsque le débat roule sur la métaphore réifiée, Erion/Olivier Paquet reproche à Lem au contraire de ne pas avoir articulé sa réflexion avec celle d'un théoricien précédent ${ }^{18}:$ «Tu veux dire que tu vas arriver aux résultats de Darko Suvin écrits... 30 ans plus tôt ? Ça aurait pas été plus rapide de commencer par Darko Suvin dès le départ? » - à quoi l'intéressé, à ce stade de la discussion, se dégage totalement du protocole académique de recherche cumulative du consensus :

Pourquoi partir de quelqu'un d'autre puisqu'il s'agit de mettre en avant la dimension subjective de l'expérience ? Pourquoi invoquer le « je » d'un tiers alors que le mien est disponible en permanence ? Je m'appuie sur ma propre relation à la SF - sur ma propre subjectivité - et j'étends progressivement les conclusions que j'en tire. (p. 278, 20 décembre 2009 20h01).

33 C'est peu dire que sa revendication de subjectivité ne convainc pas :

ce que tu présentes, à juste titre, comme ta vision personnelle du bidule, et qui a toute la légitimité voulue, tu veux en faire la définition pour tout le monde, en basant une histoire de la SF dessus (Sand, p. 278, 20 décembre 2009 20h45).

34 Au-delà de la démarche peu claire de Lehman, c'est la recherche de consensus ellemême qui cristallise le débat dans cette communauté, et par conséquent aussi les épisodes de son effort. Une preuve assez éloquente en est que durant un an de discussions qui revinrent régulièrement sur la question de la relégation culturelle de la $\mathrm{SF}$ « vue de la rue de Valois » et « vue de la rue d'Ulm » (MF, p. 621, 4 mars 2010 15h32), le fil M ne mentionne pas une seule fois le " Mois de la science-fiction à l'ENS-Ulm » ${ }^{19}$ où furent pourtant présents tous les participants du fil $\mathrm{M}$, de Gérard Klein à Georges Bormand ou Olivier Paquet, et où Lehman formula une fois de plus sa théorie de la SF.

On sait depuis Barthes ${ }^{20}$ que manier l'autorité sans jouer complètement le jeu (notamment discursif) de l'institution peut représenter un axe majeur de paratopie discursive ${ }^{21}$; lorsque Lehman s'y risque à son tour, c'est donc en affirmant son appartenance à la communauté du fandom (qu'il appelle "Notre Club»). En se souvenant de cette position de repli, on peut estimer pour le moins ambigu le geste consistant à saturer l'espace du forum avec un très long extrait d'une « conférence faite 
au 5ème colloque international de SF de Nice reprise ensuite dans les actes, puis dans Fiction " (c'est ainsi que Lehman la présente dans le forum). La section citée est la plus méthodique (et développe la question de la métaphore réifiée); les autres parties développent un propos qu'une autre conférence universitaire de Lehman titrerait encore mieux : "Pour une définition auto-théorique de la science-fiction " (Lehman, 2006b). Donnée un an après celle de Nice, dans les murs de l'ENS-Ulm, cette seconde conférence témoigne d'un projet effectivement à l'œuvre dans la carrière de Lehman au début des années 2000 (dont l'une des premières formulations, comme il le rappelle luimême, se trouve dans un article de la revue Europe en 2001: "La physique des métaphores »). Elle témoigne aussi d'une ambition qui tourne résolument le dos à la recherche d'une objectivation du consensus, c'est-à-dire du protocole savant que les colloques universitaires s'efforcent de mettre en œuvre. Le jeu paratopique de l'écrivain-théoricien Serge Lehman s'affirmait pleinement dans ces deux contextes académiques, mais sous une forme en quelque sorte inverse de celle qui s'essaye dans les semaines de novembre-décembre 2009 du fil M.

Pour moi, la fréquentation épisodique du fil M pendant l'année 2009-2010 n'a pas peu compté dans le projet ReS Futurae, alors à l'état d'ébauche. Il s'agissait de structurer un discours critique sur la SF, bien sûr, et de se donner les moyens d'éviter le double discours de la subjectivité et de l'autorité. Tel qu'on l'a vu à l'œuvre dans le fil, il empêche que le savoir se fixe, et que les hypothèses (fût-ce celle de la métaphysique ou de son déni) développent leur potentiel heuristique. Mais cela m'a également renvoyée à la question du chercheur face au fandom, plus large finalement que celle du protocole savant : comment prendre en compte le discours d'une communauté, en respectant les marques de ses caractères, que ce soit la relégation culturelle ou le programme interprétatif, c'est-à-dire sans distordre ce discours, qui les encode toujours nécessairement? Comment faire entendre l'herméneutique des amateurs? J'ai posé cette question précisément l'année du mois de la SF à l'ENS-Ulm, dans un livre et dans une $\mathrm{HDR}^{22}$; l'honnêteté implique de reconnaître qu'ils n'ont fait progresser que d'un très petit pas le front du dialogue savant / amateur. J'en donnerai un seul exemple, côté savant ${ }^{23}$. Avec un timing assez ironique, l'EHESS et le CRAL se sont consacrés à la théorie des tropes au cours d'un séminaire significativement nommé «Figure et fiction » en $2007^{24}$ (creusant la notion de métalepse, dans le sillage de la publication de Figures $V$ de Genette), moins d'un an après que Lehman a exposé sa théorie de la métaphore réifiée qui en rencontrait parfaitement le propos. Mais la séance du 28 mars 2007 où je devais présenter ses propositions tourna court. Personne dans ce séminaire n'avait lu au moins partiellement les corpus de SF dont il s'agissait; et la proposition, du plus haut intérêt, resta lettre morte au CRAL en 2007, tout comme en 2010 (dans le fil M). Le découragement peut s'inviter, quand domine l'impression que les théoriciens ne connaissent pas les corpus, et que les connaisseurs du corpus ne reconnaissent pas les théories; la revue ReS Futurae et son carnet, depuis 2012, sont nos principales actions destinées à lutter contre ce découragement.

\section{Conclusion}

Revenir sur le "Mois de la SF à l'ENS-Ulm » fut l'une des priorités de la revue ReS Futurae $\left(\mathrm{n}^{\circ} 2,2012\right)$; revenir sur le « fil M », une priorité de son carnet en ligne (Bréan, 2013). Cette répartition dit assez toute la richesse d'une position «auto-théorique » 
comme celle de Serge Lehman, qui s'est distinguée par son caractère central et persistant à l'échelle du fil ; mais qui n'est pas la seule à s'être manifestée au cours de cette discussion, Roland C. Wagner et Gérard Klein y ayant notamment aussi trouvé l'occasion d'apporter des propositions théoriques, également combattues et nuancées selon un processus de déconstruction créatrice. L'intérêt que nous portons à ces échanges et à ces réflexions s'accompagne d'un certain embarras pour des universitaires, dont les protocoles de validation diffèrent, et sont souvent inopérants dans ce type d'espace de discussion. Mais notre choix dit aussi où nous puisons l'intérêt vivant pour la SF, le foisonnement des idées de ses lecteurs, et l'irremplaçable érudition de ses amateurs : on a tâché d'expliquer ici aussi bien les raisons de nos réserves que celles de nos adhésions, en montrant comment nous avons vécu un épisode de cet intérêt passionné, et ce qu'il est devenu dans nos travaux ${ }^{25}$.

\section{BIBLIOGRAPHIE}

\section{Références critiques}

Barthes Roland, S/Z, Paris, Le Seuil, 1971.

Bréan Simon, «L'érudition de science-fiction en France : repères bibliographiques ", ReS Futurae [En ligne], 1 | 2012, mis en ligne le 02 octobre 2012, consulté le 05 mars 2018. URL : http:// journals.openedition.org/resf/179 ; DOI : 10.4000/resf.179

Bréan Simon, «Les érudits de la science-fiction en France, une tradition critique endogène ", ReS Futurae [En ligne], 1 | 2012, mis en ligne le 02 octobre 2012, consulté le 05 mars 2018. URL : http:// journals.openedition.org/resf/131; DOI : 10.4000/resf.131

Bréan Simon, «La prescription littéraire en science-fiction française ", Prescription culturelle: Avatars et métamorphoses, Brigitte Chapelain et Sylvie Ducas (dir.), Villeurbanne, Presses de l'Enssib, coll. « Papiers », 2018, p. 173-185.

Ertzscheid Olivier, « La vie en biais », Affordance.info, ISSN 2260-1856. 7 février 2017. [En ligne] https://affordance.typepad.com/mon_weblog/2017/02/vie-en-biais.html (consulté le 19 décembre 2018)

Langlet Irène, «Étudier la science-fiction en France aujourd'hui », ReS Futurae [En ligne], 1 | 2012, consulté le 30 juin 2018. URL : http://journals.openedition.org/resf/181 ; DOI : 10.4000/resf.181.

Langlet Irène, La Science-fiction. Lecture et poétique d'un genre littéraire, Paris, Armand Colin, 2006.

Maingueneau Dominique, Trouver sa place dans le champ littéraire. Paratopie et création, Louvain-laneuve : Academia, coll. « Au cœur des textes », 2016.

Westfahl Gary, «La tradition populaire de la critique de science-fiction, 1926-1980 », ReS Futurae [En ligne], 1 | 2012, mis en ligne le 02 octobre 2012, consulté le 05 mars 2018. URL : http:// journals.openedition.org/resf/225 ; DOI : 10.4000/resf.225 


\section{La controverse "Sense of Wonder et métaphysique " (2009-2010)}

Bogdanoff Igor \& Grichka, L'Effet Science-Fiction : à la recherche d'une définition, Paris, Robert

Laffont, coll. « Ailleurs et Demain », 1979.

Bréan Simon, « Retour sur la métaphysique (une introduction) », sur le carnet de Res Futurae, 14

février 2013; en ligne, URL (novembre 2017): https://resf.hypotheses.org/1559

Lehman Serge (dir.), Retour sur l'horizon, Paris, Denoël, coll. « Lunes d'Encre », 2009.

Lehman Serge (dir.), Escales sur l'horizon, Paris, Fleuve Noir, coll. « Grand Format SF », 1998.

Wagner Roland C., « Retour sur l'horizon : du Sense of Wonder à la SF métaphysique ? (1/2) », in Bifrost $\mathrm{n}^{\circ} 55$, octobre 2009 ; en ligne sur le blog du Bélial, mardi 27 octobre 2009; URL (novembre 2017) : http://blog.belial.fr/post/2009/10/27/Retour-sur-l-horizon-du-sense-of-wonder-a-la-SFmetaphysique

\section{Lehman sur la SF française et la métaphore réifiée (2001-2007)}

Lehman Serge, «La physique des métaphores », in Europe, n870, octobre 2001, p.32-50.

Lehman Serge, « La Légende du processeur d'histoire » [colloque de Nice, 2005], in Cycnos, vol. 22 $\mathrm{n}^{\circ} 1$, novembre 2006 ; en ligne, URL (novembre 2017) : http://revel.unice.fr/cycnos/index.html? $\mathrm{id}=451$

Lehman Serge, " Pour une définition auto-théorique de la science-fiction », Mois de la sciencefiction à l'ENS, 12 mai 2006, disponible en ligne, URL (juin 2018) : http://www.diffusion.ens.fr/ index.php?res=conf\&idconf $=1274$.

Lehman Serge, « Les extraterrestres, entre science et culture populaire : De la science-fiction comme laboratoire métaphysique », Le Monde diplomatique, Juillet 2009, p. 14-15; en ligne, URL : https://www.monde-diplomatique.fr/2009/07/LEHMAN/17449.

Langlet Irène, «La science-fiction, figures et fiction », communication au séminaire « De la figure à la fiction », ENS Ulm, 28 mars 2007. GDR « Fiction » CNRS-EHESS, ENS, Paris I, Paris VII, sous la direction de Jean-Marie Schaeffer. Responsables du séminaire : Michel Murat (Paris IV-ENS), Marielle Macé (CNRS-EHESS).

\section{Les espaces numériques de discussion du fandom francophone (par ordre d'apparition chronologique)}

Groupe usenet fr.rec.arts.sf, depuis 1996, URL (nov. 2017) : http://www.usenet-fr.net/fur/ chartes/rec.arts.sf.html

Liste de diffusion SF-Franco, responsable Jean-Louis Trudel (Québec), depuis 1993 ; contact : sffranco@cyberus.ca, informations sur le site Noosfere, URL (novembre 2017) : http://sf.emse.fr/ Lists.html\#SFFRANCO

Le Cafard cosmique, site web d'informations et d'opinions sur la SF, 1999-2011, site resté ouvert jusqu'en 2017, URL archive (novembre 2017) : http://archive.wikiwix.com/cache/? url=http\%3A\%2F\%2Fwww.cafardcosmique.com\%2F 
Forum ActuSF, sur le site web des éditions ActuSF, depuis 2003, URL (novembre 2017) : http:// www.actusf.com/forum/faq.php?sid=561cac023a526d3e8dc845457814ed64

\section{NOTES}

1. Pour le domaine américain, voir notamment Westfahl (2012).

2. Voir Bréan (2012a et b). Il faudrait également prendre en compte les "fanzines ", revues d'amateurs porteuses d'informations souvent précieuses, mais dont la diffusion et la conservation limitées rendent moins net l'impact global sur les connaissances partagées.

3. Serge Lehman est un écrivain et essayiste français. Figure essentielle de la science-fiction française des années 1990-2000, ayant reçu de nombreux prix pour ses romans et nouvelles, il est notamment l'auteur du cycle F.A.U.S.T. (1996-1997), d'Aucune étoile aussi lointaine (1998) et du Livre des Ombres (2005), ainsi que de la série dessinée La Brigade chimérique (avec Fabrice Colin et Gess, 2009-2010). Il a développé un ensemble d'hypothèses et de théories sur la science-fiction et l'anticipation, dans «La physique des métaphores» (2001), "La légende du processeur d'histoire » (2005), « Hypermondes perdus » (2006, préface au recueil Chasseurs de chimères), et bien sûr la préface dont il est question dans cet article.

4. Le fil de discussion s'est depuis augmenté de quelques pages en réaction à notre travail de recherche, témoignage d'un intérêt encore présent.

5. Roland C. Wagner est un écrivain et essayiste français (1960-2012). Défendant dans de nombreuses préfaces et postfaces le versant " populaire » de la science-fiction, en particulier la collection Anticipation du Fleuve Noir, il a très tôt été un membre actif du domaine des fans, le fandom, où il s'est fait connaître par son humour et sa connaissance de l'histoire de la sciencefiction. Ses romans, nouvelles et cycles, ont été récompensés par de nombreux prix. Il est notamment l'auteur des cycles de l'Histoire du Futur proche (1985-1998) et des Futurs Mystères de Paris (1996-2006), ainsi que de l'uchronie Rêves de Gloire (2011). Il a aussi participé à la fondation des Journées interdisciplinaires Sciences et Fiction de Peyresq.

6. Un récit circonstancié et une étude des arguments initiaux peuvent être lus dans Bréan (2013). 7. Outre sa qualité d'écrivain et de directeur de collection (Ailleurs et Demain, Le Livre de Poche), Gérard Klein est un essayiste ayant signé un nombre considérable de préfaces et de textes critiques témoignant d'une connaissance aiguë de la science-fiction, de son histoire et de ses enjeux (ses préfaces peuvent être notamment consultées sur le site de l'association Quarante-Deux (https://www.quarante-deux.org/les_Archives_stellaires/Gerard_Klein/). Joseph Altairac est sans doute le plus grand érudit français actuel de la science-fiction, ce dont témoignent l'encyclopédie des Terres creuses (avec Guy Costes, Encrage, 2006), et plus récemment Rétrofictions (avec Guy Costes, Encrage, 2018), une encyclopédie considérable répertoriant l'ensemble des productions culturelles liées à l'anticipation et à la science-fiction en France de Rabelais à 1951. Olivier Paquet est un écrivain dont le premier roman (Structura maxima, 2003) avait fait forte impression, et qui a depuis publié notamment le cycle du Melkine (2012-2013), salué par le Prix Verlanger en 2014; outre l'autorité de l'écrivain, il a déjà manifesté sur les forums ses connaissances en matière de science-fiction, et en particulier son attention à la culture japonaise (manga, anime, littérature).

8. Les éclats devenus quotidiens sur les réseaux sociaux atténueraient peut-être le caractère transgressif de ces débordements. Il s'agissait essentiellement d'attaques ad hominem, remettant en cause l'honnêteté intellectuelle de protagonistes. Néanmoins, le cadre de la discussion tient à cet égard plus de la querelle familiale, dont les familiarités glissent parfois jusqu'à l'injure, que des attaques que se permettent actuellement les «trolls» anonymes. Ces propos étaient strictement surveillés, voire effacés par les modérateurs, qui ont parfois fermé l'accès au fil de discussion le temps que les esprits s'apaisent. 
9. Pour une synthèse accessible, voir par exemple Ertzscheid Olivier, " La vie en biais ", Affordance.info, ISSN 2260-1856. 7 février 2017. [En ligne] https://affordance.typepad.com/ mon_weblog/2017/02/vie-en-biais.html (consulté le 19 décembre 2018)

10. Il est difficile de mettre ici tout à fait de côté mon implication subjective, aussi bien à l'époque qu'au moment où je contribue à cette réflexion. J'ai participé au fil « Du Sense of Wonder à la SF métaphysique " avec enthousiasme, car j'y trouvais l'occasion de réfléchir sur des questions essentielles pour ma thèse sur la science-fiction en France alors en cours d'achèvement, mais aussi dans l'espoir de contribuer simultanément à l'émergence d'un consensus constructif et à la réconciliation des principaux protagonistes, pour lesquels j'éprouvais de l'estime et souvent de l'amitié. (Simon Bréan)

11. Le point de départ de ce moment de la discussion peut être lu ici http://www.actusf.com/ forum/viewtopic.php?t=8050\&postdays=0\&postorder $=$ asc\&start $=6510$.

12. L'analyse de Serge Lehman a contribué de manière décisive à rendre de nouveau visible cet écrivain et surtout théoricien de l'imaginaire scientifique qu'est Maurice Renard, et dont les articles sur le «merveilleux scientifique " (1909) et le "roman d'hypothèse » (1928) sont des points de repère essentiels dans l'histoire de la littérature conjecturale française. Ces articles, ainsi que des études sur l'esthétique de Maurice Renard, peuvent être lus dans un numéro récent de ReS Futurae : dossier " Maurice Renard ", sous la direction d'Émilie Pézard et Hugues Chabot, 2018, consulté le 3 juillet 2018, URL : https://journals.openedition.org/resf/792.

13. Pendant les pages qui suivent, les discussions ne concernent pas exclusivement l'« Effet SF » : plusieurs pistes sont examinées en parallèle, dans des conversations qui n'incluent pas forcément Serge Lehman. Il faudrait ici prendre en compte plusieurs interférences polémiques, en particulier les moqueries de Roland $\mathrm{C}$. Wagner, qui à ce stade de la discussion se contente de dire que Lehman ne fait qu'habiller de grands mots des notions déjà bien connues. Même si le sarcasme et la réfutation lapidaire se manifestent ici comme ailleurs dans la discussion, il est notable que l'évocation de la métaphysique n'occasionne pas une objection de principe : ce n'est pas le caractère intrinsèquement métaphysique de la science-fiction qui est mis à l'étude.

14. L'expression employée ici de «magistère intellectuel » renvoie autant à une aspiration un peu diffuse (l'ambition de se faire un porte-parole, de fédérer autour de soi) qu'à une perception extérieure, une image imposée. Cela correspond à un mécanisme observable pendant la période de croissance de la science-fiction française, celui des " papes de la SF ». Dominique Douay écrit ainsi en 1978: "Dans la SF française, les papes sont légions, et leurs ambitions ne sont pas forcément identiques : pour un tel, il s'agira de s'instituer porte-parole de toute la SF française, pour tel autre de s'ériger en guide et censeur de l'un de ses courants " ("Le New Look de la SF française : prêt à porter ou confection ?", Fiction, n² 289, janvier-février 1978, p. 166). Toute l'ambiguïté de cette situation est qu'une initiative conçue comme constructive par son auteur peut être perçue comme " hégémonique " par les autres acteurs du champ, au risque de contrer ou de paralyser toute action.

15. Les choses ont un peu changé depuis 2010, essentiellement grâce à la revue ReS Futurae mais aussi grâce aux journées interdisciplinaires de Peyresq, séminaire annuel propice aux échanges (site web : https://sfpeyresq.sciencesconf.org/)

16. Imité de l'anglais paper, plutôt neutre en régime académique, le terme papier en français avait encore des connotations moins institutionnelles en 2009.

17. $\mathrm{mp}$ : «messages personnels» (qui ne sont pas diffusés sur le forum, mais seulement d'un participant à un autre).

18. Il s'agit de Darko Suvin, auteur d'une théorie fort productive de la SF comme «cognitive estrangement" (distanciation cognitive) (voir Pour une poétique de la science-fiction. Études en théorie et en histoire d'un genre littéraire, Québec, Presses de l'université du Québec, 1977).

19. Cet événement scientifique exceptionnel, organisé par Sylvie Allouche (univ. Paris I et Lyon

I), Simon Bréan (univ. Paris IV) et Michel Murat (Paris IV), fut l'occasion de plusieurs journées 
d'étude, d'expositions, de tables rondes, de concerts et performances. Les enregistrements sont disponibles sur le site de l'ENS : http://www.diffusion.ens.fr/index.php?res=cycles\&idcycle=280. 20. Roland Barthes, S/Z, Paris, Le Seuil, 1971.

21. Au sens de Dominique Maingueneau, Trouver sa place dans le champ littéraire. Paratopie et création, Louvain-la-neuve, Academia, coll. « Au cœur des textes », 2016.

22. Langlet Irène, La Science-fiction. Lecture et poétique d'un genre littéraire, Paris, Armand Colin, 2006 ; «La transaction littéraire. Genres, textes, savoirs », Habilitation à diriger des recherches, université de Nantes, décembre 2006.

23. Côté amateur, j'ai exprimé ce que j'en pensais dans le fanzine Remparts («A propos du fandom (Lettre d'une universitaire à un ami du fandom)», 2010/1) et dans l'article «Étudier la sciencefiction en France aujourd'hui » (2012).

24. Séminaire «De la figure à la fiction », ENS Ulm. GDR «Fiction» CNRS-EHESS, ENS, Paris I, Paris VII, sous la direction de Jean-Marie Schaeffer. Responsables du séminaire: Michel Murat (Paris IV-ENS), Marielle Macé (CNRS-EHESS).

25. Outre les articles sur l'érudition de la science-fiction déjà mentionnés, ces réflexions suscitées par la fréquentation du fandom et des forums interviennent dans «La prescription littéraire en science-fiction française » (Bréan, 2018).

\section{RÉSUMÉS}

L'érudition des fans de science-fiction s'est vigoureusement exprimée dans le champ littéraire français, dans la revue Fiction d'abord, puis dans des encyclopédies, et enfin sur des supports numériques tels que le forum d'ActuSF. C'est à partir de ce dernier que nous proposons de réfléchir à l'érudition des amateurs en science-fiction, en particulier en nous fondant sur le cas exceptionnel d'un fil de discussion alimenté entre le 28 octobre 2009 et le 17 octobre 2010, intitulé « Du Sense of Wonder à la SF métaphysique ». Ce fil de discussion a attiré de nombreux intervenants, souvent des érudits majeurs, mais de toute façon des fans et connaisseurs. L'ironie et la provocation n'y ont pas tout à fait pris le dessus sur l'argumentation et la démonstration, si bien qu'il représente un modèle remarquable de la réflexion collective en science-fiction.

\section{INDEX}

Mots-clés : science-fiction, fandom, forum, internet, ActuSF, métaphysique, Serge Lehman, Roland C. Wagner, France, sense of wonder, XXIè siècle

\section{AUTEURS}

\section{SIMON BRÉAN}

Maitre de conférences en littérature française à l'université Sorbonne Université. Auteur de La Science-fiction en France, Théorie et histoire d'une littérature (Presses Universitaires Paris-Sorbonne, 2012). Membre du comité de rédaction de ReS Futurae (https://journals.openedition.org/resf/). 


\section{IRÈNE LANGLET}

Professeure de littérature contemporaine à l'université de Paris Est Marne-la- Vallée (UPEM). A publié notamment La science-fiction. Lecture et poétique d'un genre littéraire (Armand Colin, 2006), ainsi que de nombreux articles sur ce genre. Directrice de la revue ReS Futurae (https:// journals.openedition.org/resf/). 